\title{
Development of an ultrasound acoustic streaming actuator for flow control
}

\author{
Yoshitsugu NAKA*, Kento INOUE* and Takumi ISHIZAKA* \\ * Department of Mechanical Engineering, Meiji University \\ 1-1-1, Higashimita, Tama-ku, Kawasaki, Kanagawa, 214-8571, Japan \\ E-mail:naka@meiji.ac.jp
}

Received: 27 August 2019; Revised: 1 November 2019; Accepted: 17 December 2019

\begin{abstract}
The present study aims to develop an ultrasound acoustic streaming actuator for flow control. The driving force can be derived from the continuity equation and the Navier-Stokes equation for the viscous compressible flow. Commercially available transducers are used as an ultra sound source, and the acoustic and induced flow characteristics for a single and multiple transducer configurations are examined. The sound pressure distribution indicates the strong acoustic pressure fluctuation near the transducer. For the multiple transducer cases, the region of the strong pressure fluctuation is widened due to the superposition of the waves. The distributions of the induced velocity are evaluated using particle image velocimetry. It is revealed that the maximum flow velocity is about $0.04 \mathrm{~m} / \mathrm{s}$ for the single transducer case, and the maximum velocity is observed slightly downstream of the high sound intensity region. Since the driving force is proportional to the square of the sound pressure intensity, the higher flow velocity can be achieved using more transducers. A transducer array having 100 transducers has been applied in a turbulent boundary layer. It is confirmed that the flow velocity near the wall increases in the case with the control, and turbulence intensity augments by approximately $17 \%$ compared with the case without the control.
\end{abstract}

Keywords : Acoustic streaming, Ultrasound transducers, Particle image velocimetry, Turbulent flow control, Boundary layer

\section{Introduction}

Flow control has been widely studied aiming for better performance in engineering applications: drag reduction, energy saving and noise suppression. The active flow control is considered to be a promising technology which realizes higher performance compared to passive flow treatment. Theoretical analyses of the flow control and their integrations with control theory have been developed, and their validity has been demonstrated by numerical simulations (Fukagata et al., 2002; Kim and Bewley, 2007). When applying such knowledge in practice, however, the key element is to have good sensors and actuators applicable to turbulent flows. Ideal flow actuators should have characteristics like: small in size, short response time, high efficiency and sufficient induced velocity. Though much effort has been devoted to develop actuators for active flow control (Gad-el Hak, 2006; Cattafesta III and Sheplak, 2011), it is still demanded to pursue the improvements in their performance. Here in this paper, we propose a new flow actuator using acoustic streaming.

Acoustic streaming is a steady flow driven by the sound wave in viscous fluid. The phenomenon of acoustic streaming was first found by resonance in a tube (Faraday, 1831). A net flow associated with a vibrating plate was observed, noting that currents of air ascended at displacement antinodes and descended at the nodes of the plate (Rayleigh, 1884). The flow in the inner boundary layer was also explained by Schlichting in 1932; the original paper was published in German, but the description is found in his book (Schlichting and Gersten, 2016). Eckart (1948) reported another type of streaming driven by the attenuation of the sound wave. These three types Rayleigh, Schlichting and Eckart streaming are known as the representative types of acoustic streaming, and well explained in some review papers (Lighthill, 1978; Boluriaan and Morris, 2003) In this paper, Eckart streaming is mainly considered, and the theoretical basis of the Eckart streaming is explained in Section 2. 
The acoustic streaming induced by ultrasound beam has also been extensively studied. There are several advantages in using ultrasound: directivity, inaudibleness, and high sound pressure level. Kamakura et al. (1995) studied the acoustic streaming induced by the focused Gaussian beams using numerical simulation. The experimental data have been provided in Kamakura et al. (1996), and it has been reported that the measurements show a good agreement with the numerical simulation. In addition, it has been shown experimentally that the acoustic nonlinearity enhances streaming velocity (Mitome et al., 1995). In more recent years, the effects of the driving frequency on the acoustic streaming was investigated (Dentry et al., 2014). The scaling of the acoustic streaming velocity has been formulated (Moudjed et al., 2015). The stability of the induced flow has been evaluated (Moudjed et al., 2014). It has been reported that the massively arrayed pressure transducers used for haptic device can produce 2-3 m/s acoustic streaming in the air (Hasegawa et al., 2017). Moreover, the same authors achieved a curved acceleration path in the air with sophisticated phase adjustment using a similar hardware (Hasegawa et al., 2019). Phased array ultrasound transducers can focus acoustic beams at an arbitrary location. This will provide very flexible flow actuation since the acoustic pressure fluctuation level is directly related to the magnitude of the flow driving force, and it would be an unique feature as compared to the conventional flow control actuators. However, although the basic characteristics of the ultrasound acoustic streaming has been studied, the applicability of acoustic streaming toward turbulent flow control has not yet been confirmed.

In the present study, a novel ultrasound acoustic streaming actuator for turbulent flow control is proposed. The turbulent flow control in incompressible low-speed flows is considered. The acoustic and flow characteristics of the actuator are quantified. Several ultrasound transducers are arrayed and their performance is evaluated. Acoustic and velocity characteristics have been evaluated using a reference microphone and particle image velocimetry (PIV), respectively. An ultrasound transducer array has been applied to flow control in a flat plate turbulent boundary layer.

\section{Driving force of the acoustic streaming}

The driving force of the acoustic streaming can be derived from the continuity equation and the Navier-Stokes equation of viscous compressible flow,

$$
\begin{aligned}
& \frac{\partial \rho}{\partial t}+\frac{\partial\left(\rho u_{i}\right)}{\partial x_{i}}=0 \\
& \frac{\partial\left(\rho u_{i}\right)}{\partial t}+\frac{\partial\left(\rho u_{i} u_{j}\right)}{\partial x_{j}}=-\frac{\partial p}{\partial x_{i}}+\mu \frac{\partial^{2} u_{i}}{\partial x_{j}^{2}}+\left(\frac{\mu}{3}+\mu_{B}\right) \frac{\partial}{\partial x_{i}}\left(\frac{\partial u_{j}}{\partial x_{j}}\right),
\end{aligned}
$$

where $\mu$ is the shear viscosity and $\mu_{B}$ is the bulk viscosity. The instantaneous quantity can be decomposed into the macro flow part and sound perturbed part, and therefore, the following decomposition can be applied to the density $\rho$, the pressure $p$ and the velocity components $u_{i}$,

$$
\rho=\rho_{0}+\rho^{\prime}, \quad p=P+p^{\prime}, \quad u_{i}=U_{i}+u_{i}^{\prime} .
$$

where $\rho_{0}, P$ and $U_{i}$ are the density, pressure and velocity in macro scale, and the quantities with prime denote the perturbed components. After some derivation, the driving force term $F$ appears in the governing equation for the macro flow as

$$
F=-\rho_{0} \frac{\partial \overline{u_{i}^{\prime} u_{j}^{\prime}}}{\partial x_{i}}=-\rho_{0}\left(\overline{u_{i}^{\prime} \frac{\partial u_{j}^{\prime}}{\partial x_{j}}}+\overline{u_{i}^{\prime} \frac{\partial u_{j}^{\prime}}{\partial x_{i}}}\right) .
$$

Here, overbar stands for the time averaging operation. Therefore, the mean flow is driven by the term similar to the Reynolds stress. The expression of driving force can further be simplified to

$$
F \simeq \frac{2 \alpha}{c_{0}} I
$$

where $I$ is the acoustic intensity and $c_{0}$ is the speed of sound. $\alpha$ is the sound attenuation coefficient and can be expressed as

$$
\alpha=\frac{\beta \omega^{2}}{2 c_{0}^{3}},
$$

where $\omega$ is the angular frequency, and $\beta$ is the parameter related to sound attenuation given as follows,

$$
\beta=v\left(\frac{4}{3}+\frac{\mu_{B}}{\mu}+\frac{\gamma-1}{P r}\right),
$$



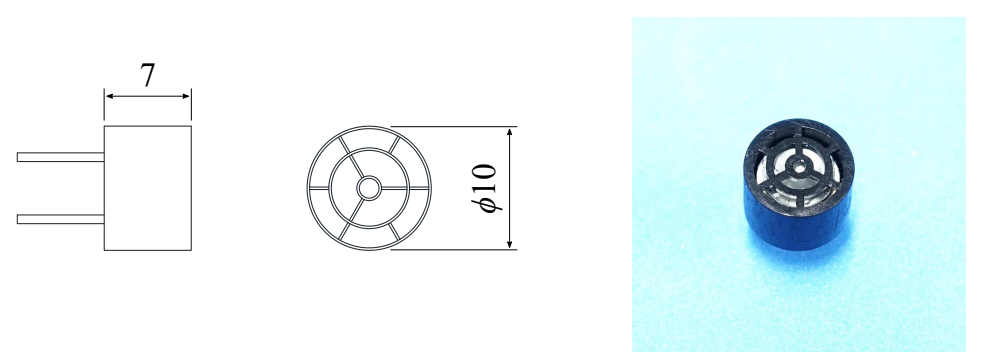

Fig. 1 The schematic and photo of an ultrasound transducer (dimensions in $\mathrm{mm}$ ).

(a)
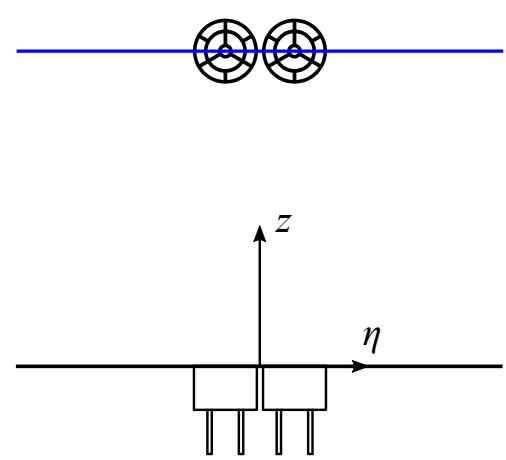

(b)
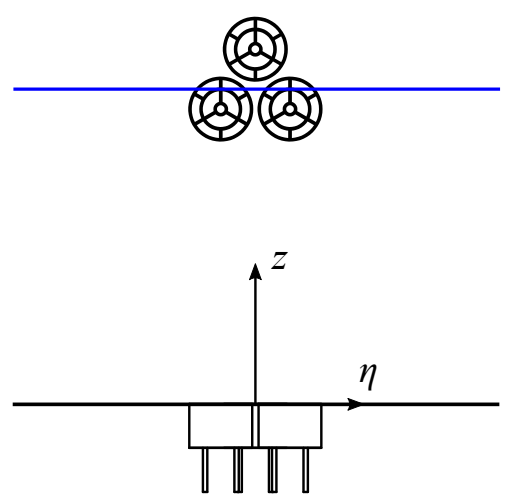

Fig. 2 Arrangement of the transducers; (a) two transducers and (b) three transducers.

where $v$ is the kinematic viscosity, $\gamma$ is the specific heat ratio and $P r$ is the Prandtl number. Based on the assumption of the plane wave and constant bulk density $\rho_{0}$, the acoustic intensity can be approximated by the square of the sound pressure fluctuation,

$$
I=\overline{p^{\prime} u^{\prime}} \simeq \frac{\overline{p^{\prime 2}}}{\rho_{0} c_{0}} .
$$

Under this assumption, the fluid driving force per unit volume can be estimated by measuring the sound pressure fluctuation. Derivation of the driving force of the acoustic streaming is well documented in Kamakura et al. (1995).

\section{Experimental setup}

\subsection{Ultrasound transducer and its arrangement}

Commercially available ultrasound transducer (UT1007-2325R, SPL Limited) has been used to generate ultrasound beam in the air. The schematic and photo of the transducer are shown in Fig. 1. The diameter and the height of the transducer are $10 \mathrm{~mm}$ and $7 \mathrm{~mm}$, respectively. The transducer generates $116 \mathrm{dBSPL}$ at the distance of $30 \mathrm{~cm}$ with the driving voltage of $10 \mathrm{Vrms}$. Here, it has been driven by the square-waveform of $\pm 15 \mathrm{~V}$ at the resonance frequency of $40 \mathrm{kHz}$, which is supplied from an in-house made oscillator and amplifier.

In the present study, single and multiple transducer configurations have been tested. The arrangements of two or three transducers are depicted in Fig. 2. The clearance between transducers is set to be minimum, which is $1 \mathrm{~mm}$ so that the effect of multiple transducers can be maximized. The measurements have been performed in the plane indicated by a blue line. For three transducer cases, the measurement plane passes through the center of the array.

These transducers create an acoustic field and also the driving force of the acoustic streaming represented by Eq. (5). This force yields a jet like streaming toward the direction of the acoustic beam propagation.

\subsection{Acoustic measurements}

The distribution of the sound pressure fluctuation was measured using a microphone (4938-A-011, B\&K) and conditioning amplifier (2690-A, B\&K). The microphone data were acquired by a 16-bit A/D converter (PCle-6361 and BNC-2110, National Instruments). The $r-\theta-z$ radial coordinate system was defined at the center of top surface of the transducer. The beam is transmitted in the axial $(z)$ direction. The measurement was performed in the rectangular region shown in Fig. 3. For convenience, the coordinate $\eta$ is defined in the $r$ direction which covers both sides of the transducer. The microphone was traversed by $2 \mathrm{~mm}$ in $\eta$ direction and $10 \mathrm{~mm}$ in $z$ direction. The measurement covers the region with 


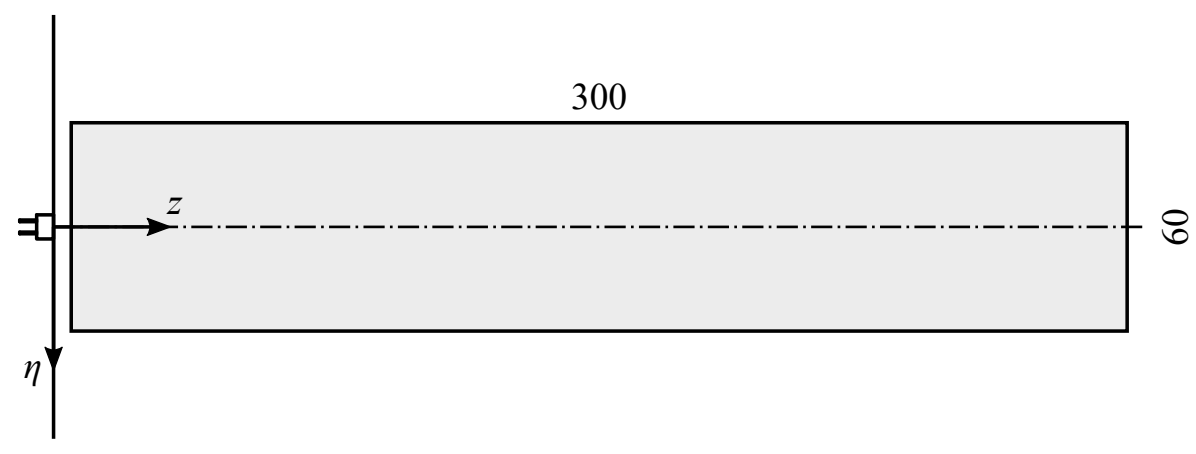

Fig. 3 Measurement region of the sound field (dimensions in $\mathrm{mm}$ ).

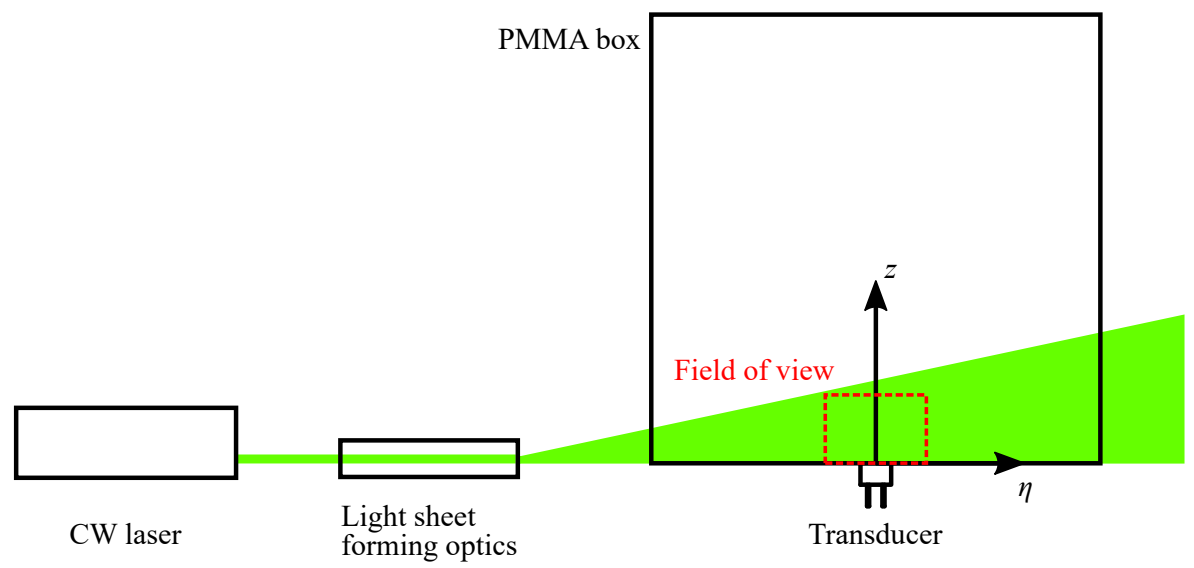

Fig. 4 Schematic of the velocity measurement.

$300 \mathrm{~mm}$ and $60 \mathrm{~mm}$ in $z$ and $\eta$ directions, respectively. The two-axes traverser composed of the ball screws and stepping motors with the positioning resolution of $10 \mu \mathrm{m}$ was employed for moving the microphone. The sampling frequency was set to $400 \mathrm{kHz}$, and the sampling time at each measurement point was set to $0.2 \mathrm{~s}$, which yields 2000 cycles of the ultrasound wave.

\subsection{Velocity measurements using particle image velocimetry}

The velocity field induced by the ultrasound transducer has been quantified by the particle image velocimetry (PIV). The setup of PIV is shown in Fig. 4. The transducer was fixed on the center of the bottom wall, and the surrounding air was confined by the box with the poly(methyl methacrylate), PMMA, cube with $300 \mathrm{~mm} \times 300 \mathrm{~mm} \times 300 \mathrm{~mm}$. The thickness of the PMMA cube wall is $3.0 \mathrm{~mm}$. The tracer particles of $0.3-1.0 \mu \mathrm{m}$ generated by a particle generator (Model 8304, Kanomax) were introduced from the bottom part. A laser beam from a continuous wave laser with the output power of $5 \mathrm{~W}$ (MGL-N-532, CNI) was shaped to a planar sheet parallel to $\eta-z$ plane passing through the center of the transducer. The thickness of the light sheet was adjusted to approximately $1.0 \mathrm{~mm}$ with the combination of plano convex-concave lens system. For the cases of two or three transducers, the measurement was performed in the plane shown in Fig. 2.

Images of particles were recorded using a high-speed camera (K4, $1280 \times 1024$ pixels, Kato Koken) with a $35 \mathrm{~mm}$ F\#1.4 lens (Samyang 35mm F1.4 AS UMC). The field of view was set to $65 \mathrm{~mm} \times 52 \mathrm{~mm}$ giving the pixel resolution of approximately $51 \mu \mathrm{m}$. The frame rate was determined to $100 \mathrm{~Hz}$ which is enough to capture the flow in the present case. The exposure time was adjusted to $1 / 500 \mathrm{~s}$ to obtain the clear circular image of the particles.

The flow field is quantified by two dimensional two components (2D2C) PIV. The cross correlation between two consecutive frames has been obtained through the fast Fourier transform (FFT). The interrogation window is recursively shrunk so that the good spatial resolution can be obtained. In the iterative process, the interrogation windows are shifted based on the displacement of the previous pass. The windows of both frames are shifted symmetrically by integer pixels. The sub-pixel displacement is determined with the three point Gaussian subpixel interpolation. Outliers have been identified by the method proposed by Westerweel and Scarano (2005) with the standard thresholding parameter. Outliers were replaced by cubic interpolation of valid data. The procedure described above is quite standard nowadays and it is implemented by in-house Matlab codes in reference to books (Adrian and Westerweel, 2011; Raffel et al., 2018). 


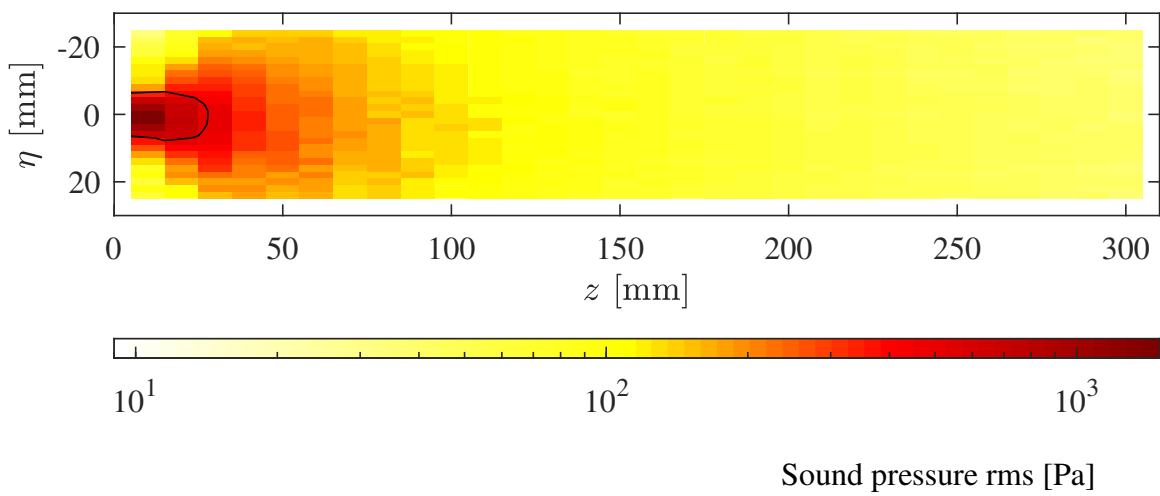

Fig. 5 Sound pressure distribution for a single transducer case.

(a)

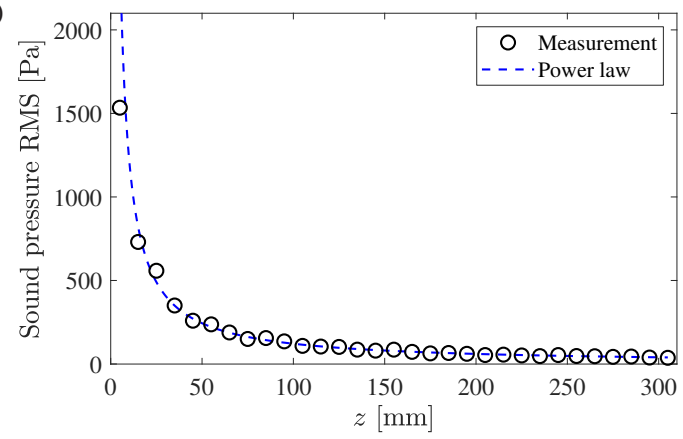

(b)

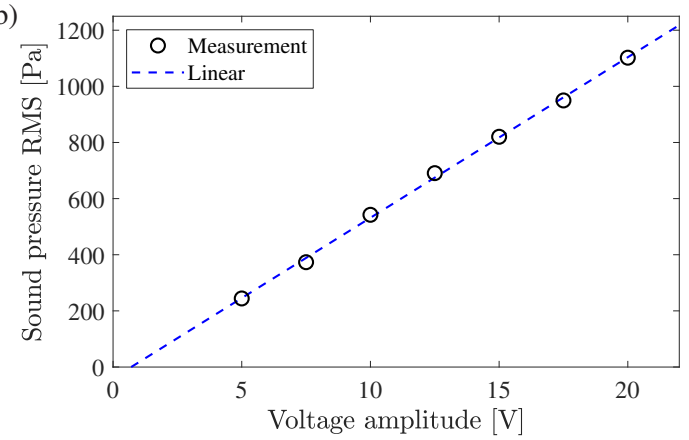

Fig. 6 Profile of the pressure fluctuation along the transducer axis (a); sound pressure against the driving voltage at $z=10 \mathrm{~mm}$ (b).

For the present analyses, the interrogation window with the size of $64 \times 64 \mathrm{px}$ was used for the first pass, and $32 \times 32$ $\mathrm{px}$ for the second, $16 \times 16 \mathrm{px}$ for the final pass. The interrogation window size of $16 \mathrm{px}$ gives the spatial resolution of approximately $0.82 \mathrm{~mm}$. The spacing between adjacent vectors are set to $16 \mathrm{px}$, which gives no window overlap. The average ratios of the outliers in different PIV conditions are $1.6 \%$. The calibration grid was recorded for the conversion of the image and physical coordinate.

3.3.1. Effect of acoustic radiation force on tracer particles The acoustic beam can directly exert the force to objects through the acoustic radiation force. It is caused by the difference in the acoustic impedance. Slama et al. (2017) discussed the effect of the radiation force depending on the particle size in PIV in water. When the particle size is large, the particles are affected by the radiation force. Here, it is important to ensure that the tracer particle is moved by the flow, not directly driven by the acoustic beam. The acoustic radiation force, $F_{\text {rad }}$, which acts on the sphere whose radius of $a$ placed in the acoustic plane progressive wave of the wave number $\left(k=2 \pi f / c_{0}\right)$ can be estimated by the following equation derived by King (1934).

$$
\frac{F_{\mathrm{rad}}}{\pi a^{2}}=4(k a)^{4} Y_{p} \bar{E}
$$

where $\bar{E}$ is the mean total energy-density in the wave, $Y_{p}$ is the non-dimensional relative density factor.

$$
Y_{p}=\frac{1+\frac{2}{9}\left(1-\rho_{0} / \rho_{d}\right)^{2}}{\left(2+\rho_{0} / \rho_{d}\right)^{2}}
$$

where $\rho_{d}$ is the density of the tracer droplet, $\rho_{0}$ is the density of the bulk ambient air. Here, the tracer particles are from the glycol solution, and the density of the liquid is estimated to be $1016 \mathrm{~kg} / \mathrm{m}^{3}$. In the present experimental condition, the mean particle radius is approximately $0.5 \mu \mathrm{m}$; density of the air is $1.183 \mathrm{~kg} / \mathrm{m}^{3} ; E$ is obtained by $\overline{p^{\prime 2}} /\left(2 \rho_{0} c_{0}^{2}\right)$; the speed of sound $c_{0}$ is the $346.3 \mathrm{~m} / \mathrm{s}$ and the frequency of the ultrasound is $40 \mathrm{kHz}$. The assumption of the above equation is $k a \ll 1$, and in the present case, it holds with $k a=3.629 \times 10^{-4}$. The value of $Y_{p}$ can be obtained as, $Y_{p}=2.116 \times 10^{-14}$. Then, the acoustic force $F_{\text {rad }}=1.318 \times 10^{-25} \mathrm{~N}$, which is negligibly small. 
(a)

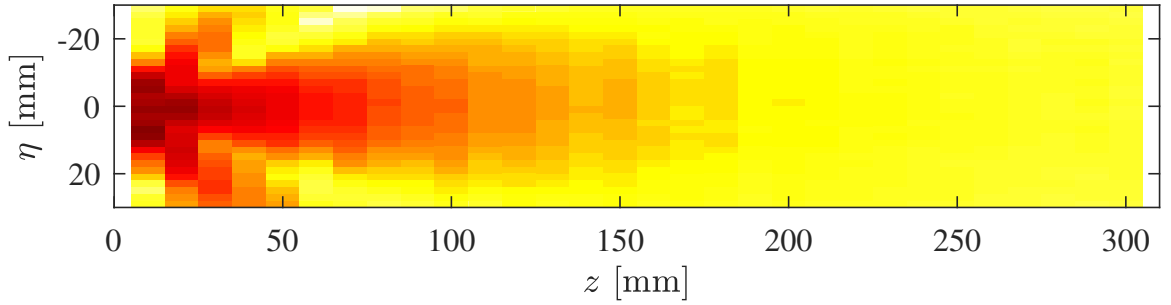

(b)
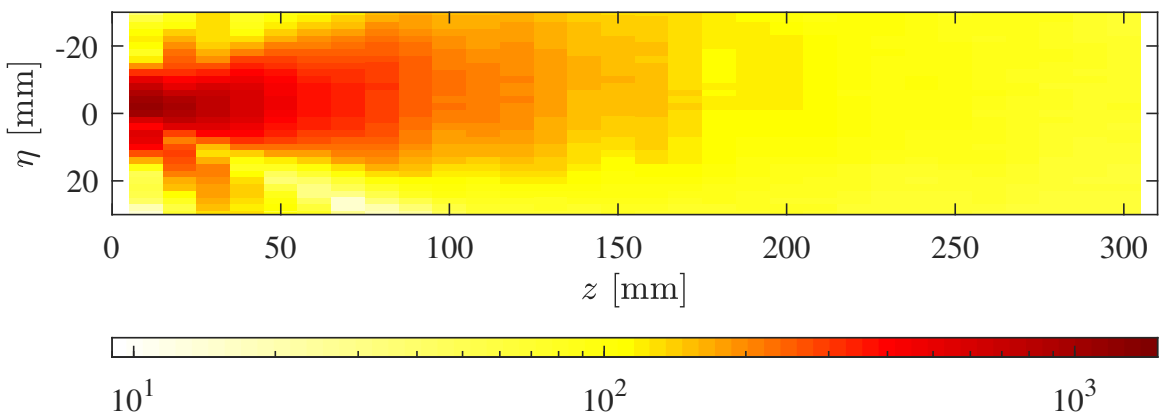

$10^{3}$

Sound pressure rms $[\mathrm{Pa}]$

Fig. 7 Sound pressure distributions for multiple transducer cases; (a) two transducers and (b) three transducers.
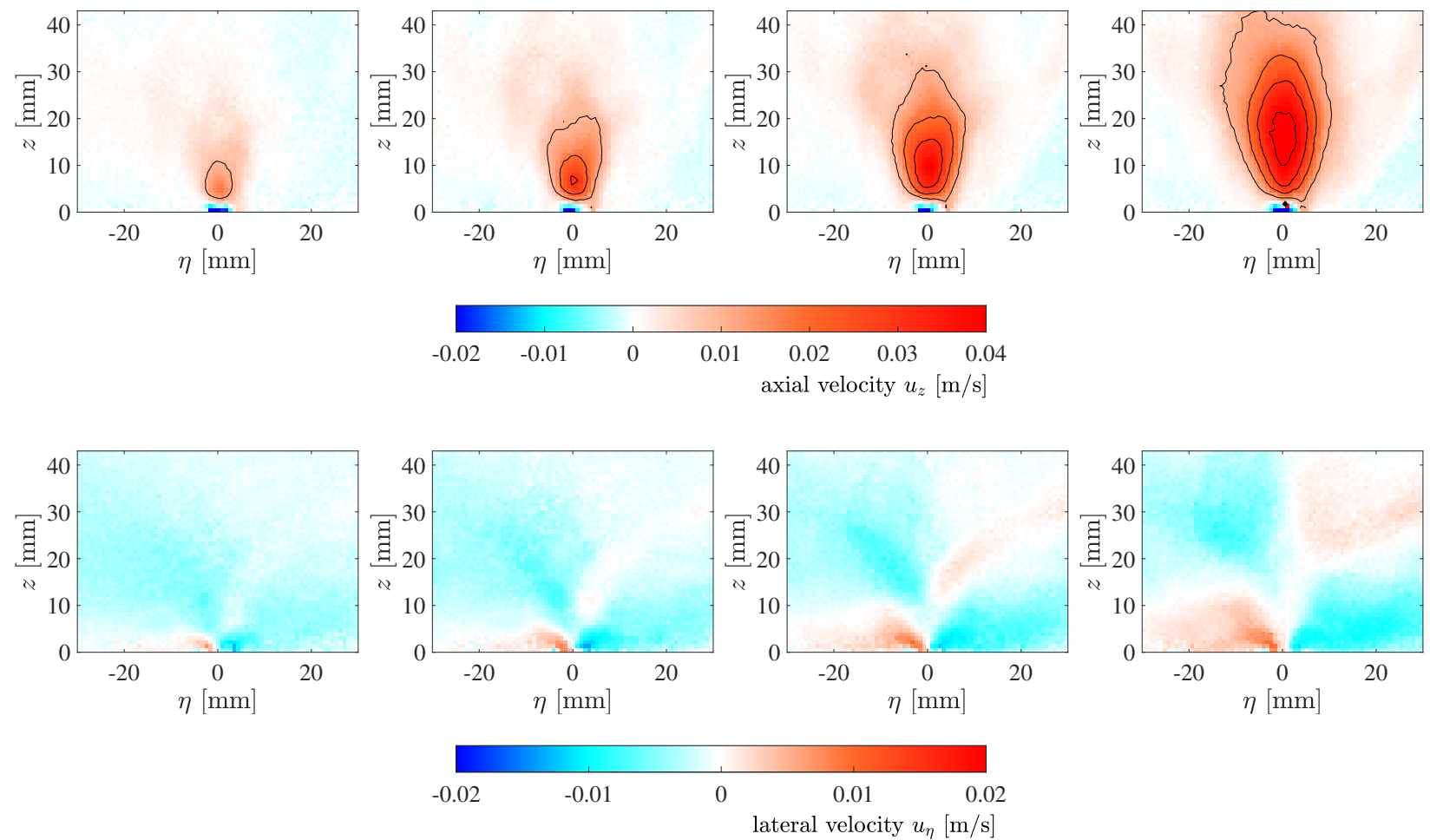

Fig. 8 Transient velocity distributions for a single transducer case at $t=0.1 \mathrm{~s}, 0.25 \mathrm{~s}, 0.5 \mathrm{~s}$ and $1.0 \mathrm{~s}$ (from left to right); axial velocity component $u_{z}$ (top), lateral velocity component $u_{\eta}$ (bottom).

\section{Acoustic characteristics}

The distribution of the root mean square (rms) values of sound pressure fluctuation in the $\eta-z$ plane is shown in Fig. 5. It mostly indicates an axi-symmetric distribution. For the single transducer case, the high sound intensity region is relatively restricted in the near field of the transducer. The region where the acoustic intensity is higher than $500 \mathrm{~Pa}$ is indicated by a solid line and expands $12.7 \mathrm{~mm} \times 27.8 \mathrm{~mm}$ in $\eta$ and $z$ directions, respectively. The peak of the sound pressure level is $1534 \mathrm{~Pa}$ and it is observed at the closest measurement point. The expected driving force per volume $F$ is $1.00 \mathrm{~N} / \mathrm{m}^{3}$ according to Eq. (5). 

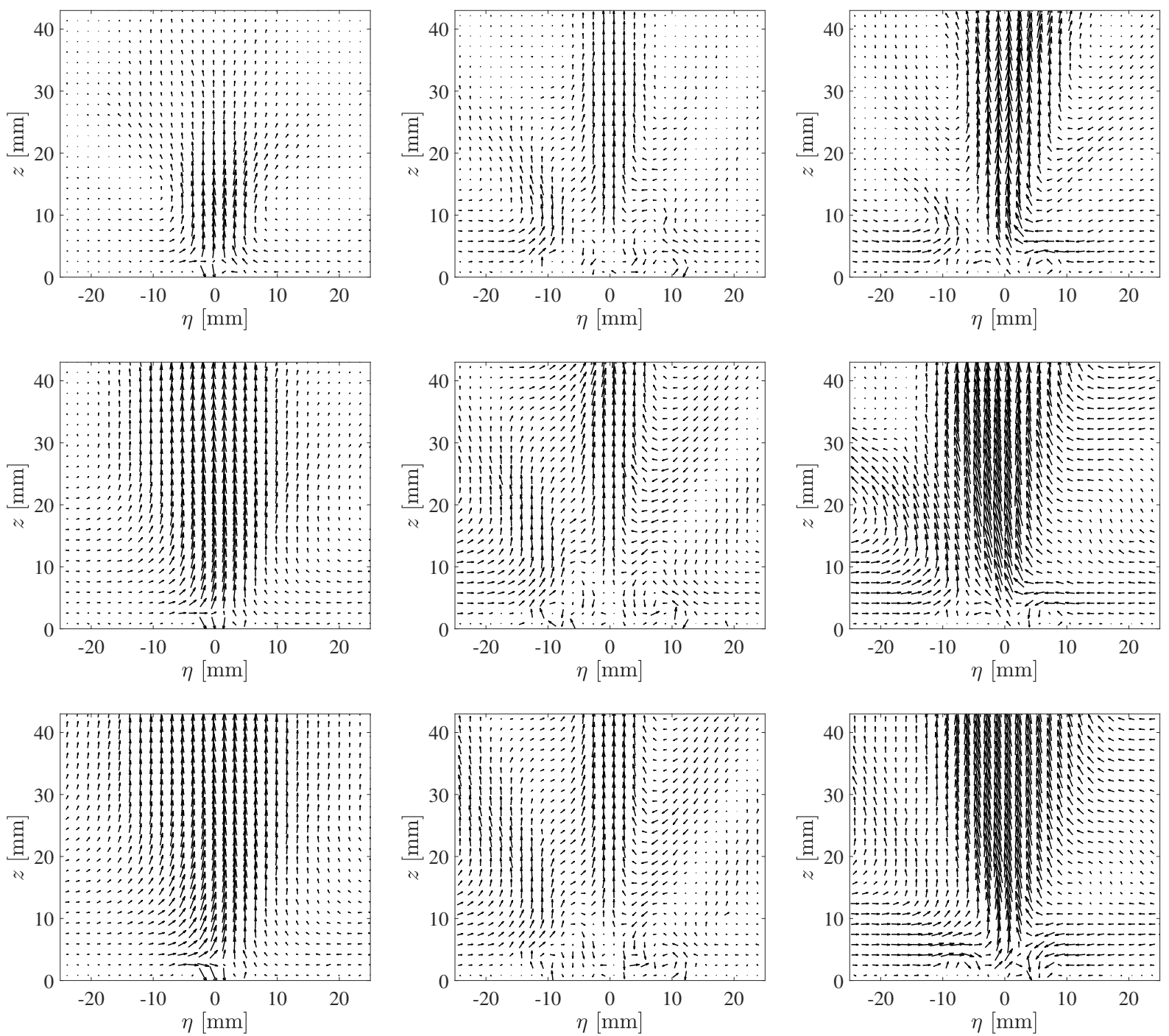

Fig. 9 Distributions of velocity vectors for single (left), double (center) and triple (right) transducer configurations at $t=0.5 \mathrm{~s}$ (top), $2.0 \mathrm{~s}$ (middle) and $5.0 \mathrm{~s}$ (bottom).

Figure 6(a) indicates the profile of the pressure level along the transducer axis. It decreases with the inverse of the distance from the transducer, which is the weaker slope anticipated from the point source ( $r^{-2}$ attenuation). This is because the wave can be considered as the near field ultrasound, and it can be treated as the quasi-plane wave. Figure 6(b) shows the rms values of sound pressure against the driving voltage amplitude. The sound pressure rms value increases linearly with the input voltage amplitude. In the present study, the driving voltage is fixed to $15 \mathrm{~V}$.

The distributions of the sound pressure level for two and three transducer cases are presented in Fig. 7. The distribution of the sound pressure level is somewhat different from the single transducer case. They indicate wider region of high sound pressure, and the development of side lobe is visible. It can be explained by the superposition of the waves. The sound pressure distribution extends from the transducers in three directions. As the number of transducers increase, the area of the high sound intensity region grows. In the case of the three transducers, though the measurement plane is located slightly off from the transducer axis, the high sound intensity region is observed in the broader region. Since the induced velocity of acoustic streaming is the function of the sound pressure intensity, the present results indicate the stronger flow can be achieved with more transducers taking advantage of the sound superposition principle.

\section{PIV measurement of the induced flow}

The velocity distributions for a single transducer case are shown in Fig. 8. The flow is accelerated by the driving force of acoustic streaming, and it develops in time, and it mostly achieves steady state after $1.0 \mathrm{~s}$. The maximum velocity 


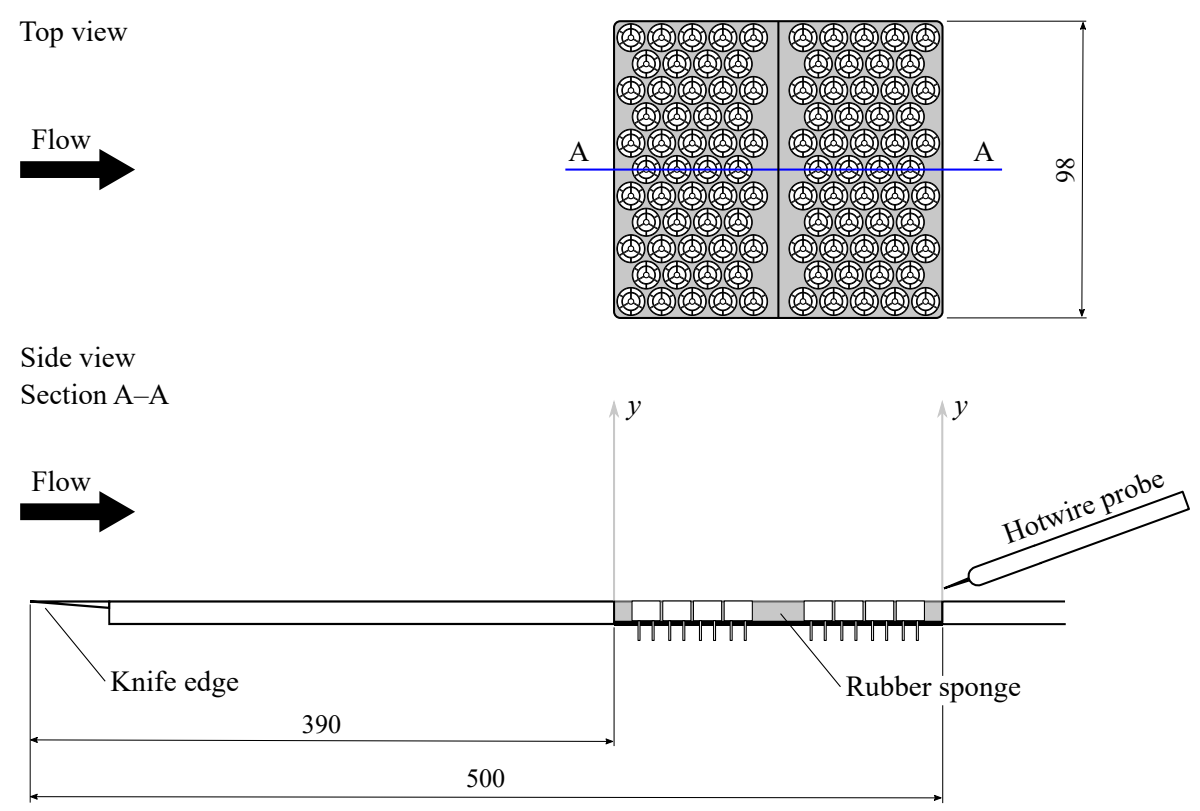

Fig. 10 Schematic of the experimental setup in a boundary layer. Dimensions are in $\mathrm{mm}$.

is observed slightly downstream from the high sound intensity region. The width of the acoustic streaming expands in the downstream direction. One second after turning on the actuator, the maximum flow velocity is $0.04 \mathrm{~m} / \mathrm{s}$. The maximum flow velocity is observed at $z \simeq 15 \mathrm{~mm}$. In Fig. 8, the flow in the radial direction is also observed. As the jet-like flow develops, the surrounding fluid is entrained toward the actuator near the wall and the jet expands in radial direction in the downstream region.

Figure 9 shows the flow velocity for different transducer configurations. Since the driving force is proportional to the square of the sound pressure intensity, the higher flow velocity can be achieved using more transducers. For the multiple transducer cases, the distributions of driving force are expected similar to the sound pressure as presented in Fig. 7. For the case with three transducers, the axial flow is prominent, and side lobe also induces the flow. The maximum axial velocity is $0.066 \mathrm{~m} / \mathrm{s}$ achieved at $\eta=-0.21 \mathrm{~mm}$ and $z=24.8 \mathrm{~mm}$. In addition, the vortex like flow pattern is observed close to the wall: it is most apparent in $\eta \simeq 7 \mathrm{~mm}$ and $z \leq 5 \mathrm{~mm}$. The most of the flow observed in the present experiment is the Eckart streaming where the jet-like flow is formed due to the driving force inherent to the sound absorption. However, in the region close to the wall, it is possible that the different type of acoustic streaming becomes significant. Such streaming phenomena near the wall may be of the interest in our future work. In addition, it is demonstrated that this actuator can generate various flow pattern depending on the acoustic field. The flexibility will be much more enhanced when the multiple transducers are driven with the phase difference whereas they are all driven in-phase in the present study.

\section{Application to a turbulent boundary layer}

\subsection{Experiment}

The ultrasound acoustic streaming actuator has been applied to the flow control in a turbulent boundary layer. The ultrasound actuator array, which has 50 ultrasound transducers in the area of $55 \times 98 \mathrm{~mm}$, has been used, and two arrays were arranged side by side, and the long sides were put adjacent to each other. The arrangement is depicted in Fig. 10. The maximum sound pressure level achieved by this array reaches $2000 \mathrm{~Pa} \mathrm{rms}$, and the expected driving force per volume $F$ is $1.70 \mathrm{~N} / \mathrm{m}^{3}$ according to Eq. (5).

Figure 10 shows a schematic of the experimental setup. The Cartesian coordinate system is defined at the center in the spanwise direction on the leading edge of the plate. The mean stream direction is defined as $x$, the wall normal direction is $y$. The flat plate with a knife edge was settled at the exit of a blowing type wind-tunnel with the exit section of $400 \mathrm{~mm} \times 600 \mathrm{~mm}$. The free stream velocity was set to $1.0 \mathrm{~m} / \mathrm{s}$. The boundary layer develops from the knife edge. The velocity profile was measured at the position $390 \mathrm{~mm}$ from the upstream tip. This position corresponds to right downstream of the actuator array.

The velocity distribution of the turbulent boundary layer developed along the flat plate device was measured at 23 points in the wall normal direction. An I-type hot wire probe (0251R-T5, Kanomax) was placed as the wire axis corresponds to the spanwise direction, and it was operated by a constant temperature anemometer (1011, Kanomax). 
Naka, Inoue and Ishizaka, Journal of Fluid Science and Technology, Vol.15, No.1 (2020)

Table 1 Characteristics of turbulent boundary layer at upstream of actuator array.

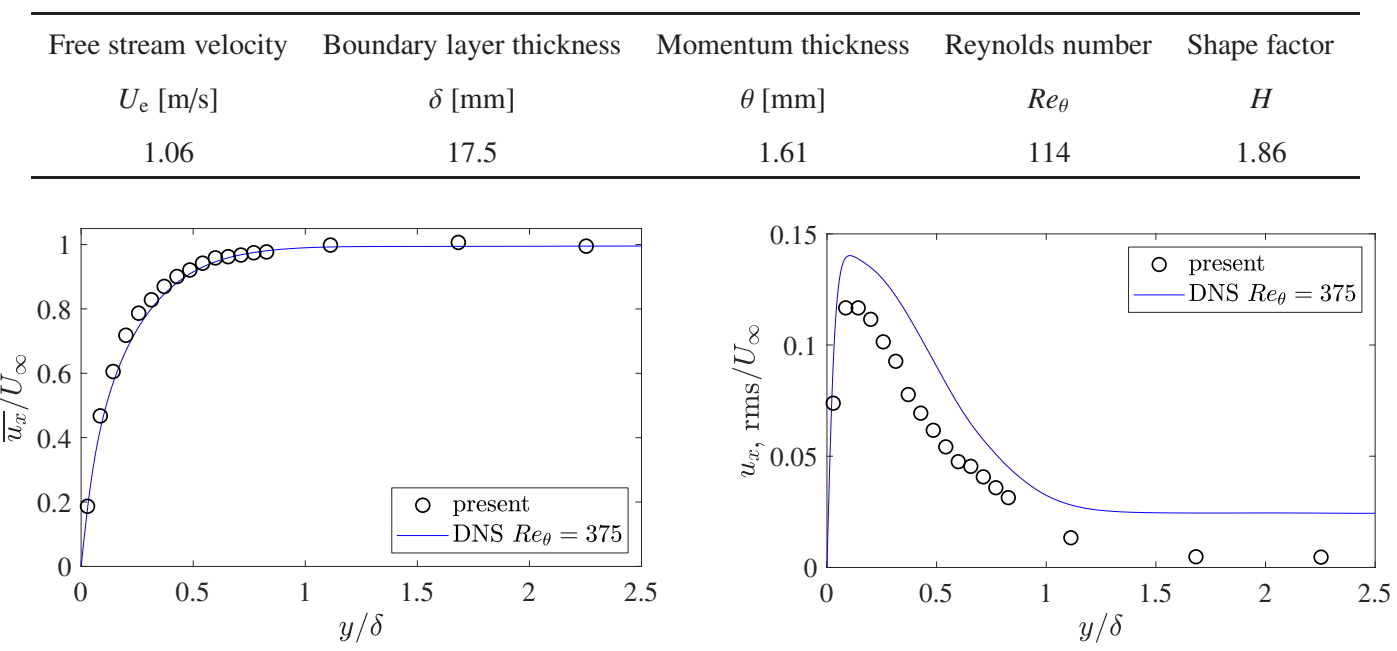

Fig. 11 Profiles of the mean velocity (left) and velocity fluctuation (right) at the upstream position. The present experimental data are compared with the DNS data of Wu and Moin (2009).
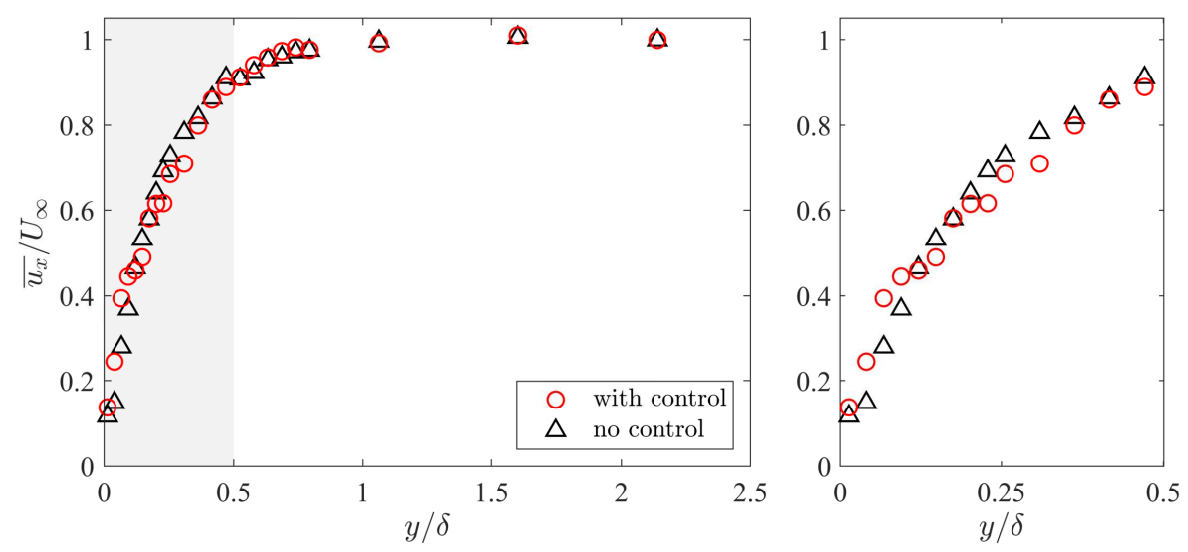

Fig. 12 Mean velocity profiles for the control on/off cases.

The velocity measurements have been undertaken immediately after the calibration of the hotwire was performed. The reference velocity for the calibration was obtained by a Pitot tube (LK-1S, Okano works) with the differential pressure transducer (DP45, Valydine and PA701, Krone). The electric signal was filtered by a passive anti aliasing low-pass filter with a cut-off frequency of $1 \mathrm{kHz}$ (EF110, Thorlabs) and acquired by A/D converter (USB-6001, National Instruments). The sampling frequency was set to $20 \mathrm{kHz}$, and the sampling time at each location was $60 \mathrm{~s}$ to ensure convergence up to second order statistics. The calibration data were fitted to a 4 th order polynomial function giving the sufficient accuracy for the present purpose Bruun (1996).

\subsection{Effect of actuator array}

In order to evaluate the characteristics of the oncoming turbulent boundary layer, the same measurements were carried out at just upstream of the transducer arrays. Characteristics of the upstream inflow boundary layer are summarized in Table 1. Though the present measurement is performed at the low Reynolds number, the shape factor at this position is 1.86 which is considerably smaller than the value of the laminar boundary layer. Therefore, it can be considered as a turbulent boundary layer.

Figure 11 shows the profiles of the mean velocity and the rms values of the streamwise velocity fluctuation at the upstream position. The mean velocity and the distance from the wall is normalized by the boundary layer thickness $\delta$ and the free stream velocity $U_{\infty}$ of the inflow boundary layer. The present experimental data are compared with the DNS data of Wu and Moin (2009). Among the available DNS data at different Reynolds number conditions, the profile at $\operatorname{Re}_{\theta}=375$ best fits the present experimental data. For the streamwise velocity fluctuations, the overall profile of the present data shows the same tendency as the DNS data. It is true that the present turbulent boundary layer is at the low Reynolds 


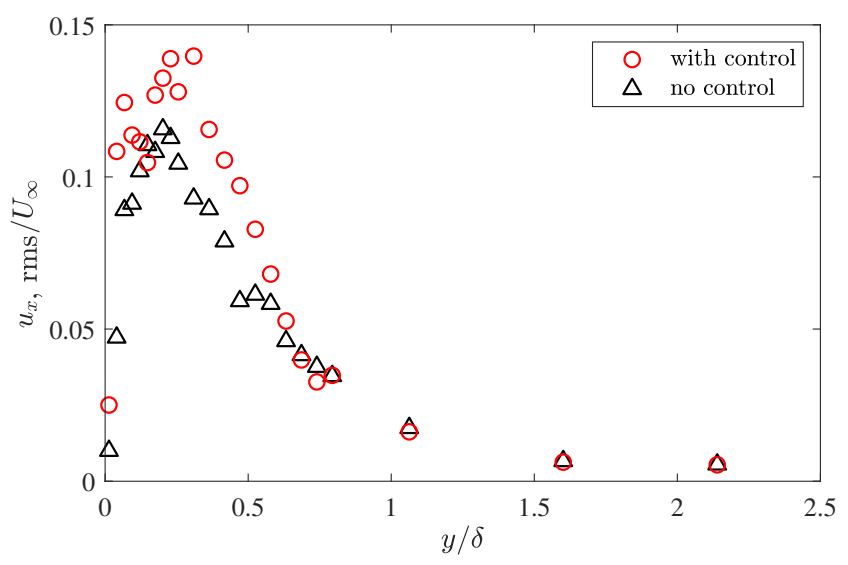

Fig. 13 Profiles of the streamwise velocity fluctuations for the control on/off cases.

number. Nevertheless, the effectiveness of the present actuator array can be examined.

At the downstream location of the actuator array, measurements were performed under the condition of actuator on or off. Figure 12 shows mean velocity profiles. When the actuator is on, the average streamwise velocity is higher near the wall than in the case with actuator off. This relationship is switched around $y / \delta=0.15$. For $0.15 \leq y / \delta \leq 0.5$, the mean velocity gradient is smaller with actuator on. Figure 13 shows profiles of turbulence intensity of streamwise velocity component. The turbulence intensity increases by approximately $17 \%$ at the peak value around $y / \delta=0.2$ compared with the case of actuator off. The effect of actuation is visible up to $y / \delta \leq 0.6$.

With this ultrasound actuator array, it is possible to influence the average velocity distribution near the wall and the distribution of the streamwise velocity fluctuations. The actuator array creates jet-like flows detaching from the wall, such flows promote ejection motions of the wall turbulence. Therefore, the activity of the near wall turbulence is enhanced and the mean velocity profile is steepened compared to the no control case. Through the present test in a boundary layer, the possibility of controlling wall turbulence by the ultrasound acoustic streaming is demonstrated.

\section{Conclusion}

A novel flow actuator for flow control has been proposed based on the ultrasound acoustic streaming. The fundamental characteristics of the ultrasound transducers have been quantitatively evaluated for single and multiple transducer cases. The distributions of the pressure fluctuation show that the driving force of the acoustic streaming can be enhanced by using multiple transducers. The flow field indicates meaningful induced velocity depending on the number of transducers. In the present experiment, a single transducer produces at most approximately $0.04 \mathrm{~m} / \mathrm{s}$ with the maximum $\mathrm{rms}$ of the pressure fluctuation of $1500 \mathrm{~Pa}$. For the multiple transducer case, the velocity pattern represents the distribution of the acoustic pressure fluctuation.

An ultra sound transducer array employing 100 elements has been applied to a flat plate boundary layer. The control effects are clearly observed on the distributions of the average velocity and the streamwise velocity fluctuations. When the control is on, the mean flow is accelerated in the near wall region and the velocity gradient is less steep further from the wall. The streamwise velocity fluctuations increases by approximately $17 \%$. The present study confirms that the ultrasound acoustic streaming can influence the wall bounded turbulent flows.

\section{Acknowledgement}

This work was partially supported by JSPS KAKENHI Grant Number JP19K04176.

\section{References}

Adrian, R. J. and Westerweel, J. Particle image velocimetry, (2011). Cambridge University Press.

Boluriaan, S. and Morris, P. J. Acoustic streaming: from Rayleigh to today. International Journal of aeroacoustics, Vol.

2, No.3, (2003), pp.255-292.

Bruun, H. H. Hot-wire anemometry: principles and signal analysis, (1996). Oxford University Press. 
Cattafesta III, L. N. and Sheplak, M. Actuators for active flow control. Annual Review of Fluid Mechanics, Vol. 43, (2011), pp.247-272.

Dentry, M. B., Yeo, L. Y. and Friend, J. R. Frequency effects on the scale and behavior of acoustic streaming. Physical Review E, Vol. 89, No.1, (2014), pp.013203.

Eckart, C. Vortices and streams caused by sound waves. Physical review, Vol. 73, No.1, (1948), pp.68.

Faraday, M. On a peculiar class of acoustical figures; and on certain forms assumed by groups of particles upon vibrating elastic surfaces. Philosophical transactions of the Royal Society of London, Vol. 121, (1831), pp.299-340.

Fukagata, K., Iwamoto, K. and Kasagi, N. Contribution of Reynolds stress distribution to the skin friction in wall-bounded flows. Physics of Fluids, Vol. 14, No.11, (2002), pp.L73-L76.

Gad-el Hak, M. Flow control: passive, active, and reactive flow management, (2006). Cambridge university press.

Hasegawa, K., Qiu, L., Noda, A., Inoue, S. and Shinoda, H. Electronically steerable ultrasound-driven long narrow air stream. Applied Physics Letters, Vol. 111, No.6, (2017), pp.064104.

Hasegawa, K., Yuki, H. and Shinoda, H. Curved acceleration path of ultrasound-driven air flow. Journal of Applied Physics, Vol. 125, No.5, (2019), pp.054902.

Kamakura, T., Matsuda, K., Kumamoto, Y. and Breazeale, M. A. Acoustic streaming induced in focused Gaussian beams. The Journal of the Acoustical Society of America, Vol. 97, No.5, (1995), pp.2740-2746.

Kamakura, T., Sudo, T., Matsuda, K. and Kumamoto, Y. Time evolution of acoustic streaming from a planar ultrasound source. The Journal of the Acoustical Society of America, Vol. 100, No.1, (1996), pp.132-138.

Kim, J. and Bewley, T. R. A linear systems approach to flow control. Annual Review of Fluid Mechanics, Vol. 39, (2007), pp.383-417.

King, L. V. On the acoustic radiation pressure on spheres. Proceedings of the Royal Society of London. Series AMathematical and Physical Sciences, Vol. 147, No.861, (1934), pp.212-240.

Lighthill, J. Acoustic streaming. Journal of sound and vibration, Vol. 61, No.3, (1978), pp.391-418.

Mitome, H., Kozuka, T. and Tuziuti, T. Effects of nonlinearity in development of acoustic streaming. Japanese journal of applied physics, Vol. 34, No.5S, (1995), pp.2584.

Moudjed, B., Botton, V., Henry, D., Millet, S., Garandet, J.-P. and Ben-Hadid, H. Oscillating acoustic streaming jet. Applied Physics Letters, Vol. 105, No.18, (2014), pp.184102.

Moudjed, B., Botton, V., Henry, D., Millet, S., Garandet, J.-P. and Hadid, H. B. Near-field acoustic streaming jet. Physical Review E, Vol. 91, No.3, (2015), pp.033011.

Raffel, M., Willert, C. E., Scarano, F., Kähler, C. J., Wereley, S. T. and Kompenhans, J. Particle image velocimetry: a practical guide, (2018). Springer.

Rayleigh, L. On the circulation of air observed in Kundt's tubes, and on some allied acoustical problems. Philosophical Transactions of the Royal Society of London, Vol. 175, (1884), pp.1-21.

Schlichting, H. and Gersten, K. Boundary-layer theory, (2016). Springer.

Slama, R. B. H., Gilles, B., Chiekh, M. B. and Béra, J.-C. PIV for the characterization of focused field induced acoustic streaming: seeding particle choice evaluation. Ultrasonics, Vol. 76, (2017), pp.217-226.

Westerweel, J. and Scarano, F. Universal outlier detection for PIV data. Experiments in fluids, Vol. 39, No.6, (2005), pp. 1096-1100.

Wu, X. and Moin, P. Direct numerical simulation of turbulence in a nominally zero-pressure-gradient flat-plate boundary layer. Journal of Fluid Mechanics, Vol. 630, (2009), pp.5-41. 